\title{
Hybrid Simultaneous Localization and Map Building: Closing the Loop with Multi-Hypotheses Tracking
}

\author{
${ }^{\dagger}$ Autonomous Systems Lab \\ Swiss Federal Institute of Technology Lausanne (EPFL) \\ CH-1015 Lausanne \\ nicola.tomatis@epfl.ch
}

Nicola Tomatis ${ }^{\dagger}$, Illah Nourbakhsh ${ }^{\dagger}$, Roland Siegwart ${ }^{\dagger}$

\begin{abstract}
In this paper simultaneous localization and map building is performed with a hybrid, metric - topological, approach. A global topological map connects local metric maps, allowing a compact environment model, which does not require global metric consistency and permits both precision and robustness. However, the most important innovation of the approach is the way how loops in the environment are handled by map building using the information of the multi hypotheses topological localization. The method uses data from a $360^{\circ}$ laser scanner to extract corners and openings for the topological approach and infinite lines for the metric method. This hybrid approach has been tested in a $50 \times 25 \mathrm{~m}^{2}$ portion of the institute building with a fully autonomous robot. The performances of the whole system are proven empirically by comparing maps generated by independent explorations, testing the localization capabilities, making relocation experiments and showing how the technique for closing the loop works.
\end{abstract}

\section{Introduction}

Research in localization and map building has recently lead to successful approaches. Most of them are mathematically well-grounded and coherent. However solutions for consistent mapping allowing precise and robust localization of the robot in unmodified and dynamic environments have not been found yet. The problem is effectively highly complex especially for simultaneous localization and map building where the robot is required to remain localized with respect to the portion of the environment that has already been mapped in order to build a coherent map.

The research on this field has diverged to different approaches which are either metric, topological or hybrid. Approaches relying on purely metric maps are vulnerable to inaccuracies in both map-making and odometry abilities of the robot. Even by taking into account all relationships between features and the robot itself, in large environments the drift in the odometry makes the global consistency of the map difficult to maintain. Topological approaches can better handle this problem, because they only have to maintain topological global consistency, not metric. However these approaches are either less precise than fully metric approaches, due to the discretization of the localization space, or computationally intractable for fully autonomous robots, when fine grained grids are used. More recently, approaches combining the metric (grid-based) and the topological paradigm have shown that positive characteristics of both world can be integrated in a hybrid method.

This paper shows how a coherent integration of the metric

\author{
\$The Robotics Institute \\ Carnegie Mellon University \\ 5000 Forbes Avenue, Pittsburgh, PA 1513 \\ illah@ri.cmu.edu
}

and topological paradigms can lead to a powerful approach for localization and map building. For this, the environmental model embodies both a metric and a topological representation. The metric model consists of infinite lines belonging to the same place. These places are related to each other by means of a topological map which is composed of landmarks (corners and openings), nodes representing topological locations and edges between nodes. Edges to a place correspond to a switch from the topological to the metric paradigm. The effectiveness of this method for localization has already been shown in [19]. In [20] the extension to simultaneous localization and map building is presented. In this paper it is shown how loops can be closed within the same framework.

For the metric approach an Extended Kalman Filter (EKF) is used. This method has already proven its strength for localization in [1]. Map building can therefore be done with the Stochastic Map approach [16]. Topological navigation uses a Partially Observable Markov Decision Process (POMDP) [4] for state estimation. This permits efficient planning in the large, has an advantageous symbolic representation for manmachine interaction and is robust due to its multi hypotheses tracking.

\section{Environment Modeling}

The environment is described by a global topological map, which permits moving in the whole environment, and local metric maps which can be used as soon as further localization precision is needed (fig. 1). In order to switch from topological to metric, a detectable metric feature is needed to determine the transition point and to initialize the metric localization. Given this transition feature, a metric place can be defined everywhere in the environment. Switching to topological does not require any specific characteristic: The robot navigates metrically to the initialization position for the current local place where it restarts its topological navigation.

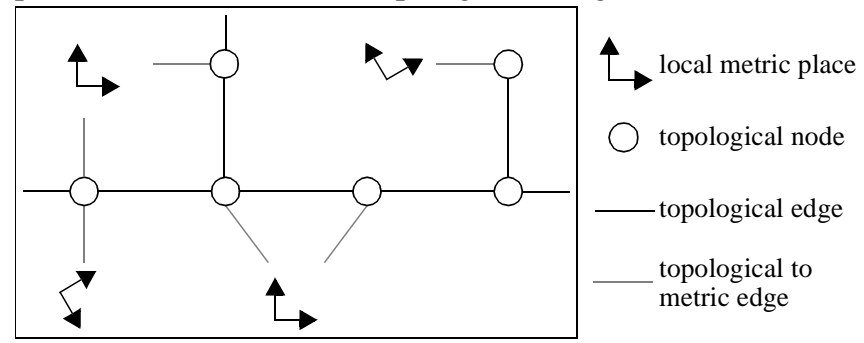

Figure 1: The environment is represented by places given by their metric maps and nodes representing topological locations. When travelling from a node to a place, the system switches from topological to metric and vice-versa. 


\subsection{Global Topological Map}

Landmarks, which are helpful for the topological model, are those permitting to distinguish between locations in the environment. In this case two different types are chosen:

- Corners, which are characterized by their orientation.

- Openings, which are also used for model transition.

The topological map can be viewed as a graph. Topological locations are represented by nodes containing the information about the way to reach the connected topological location/ metric place. Furthermore the list of the landmarks lying between two locations is represented as a list between the two nodes. In fig. 2 the graph representing the topological model is viewed for a portion of the environment.
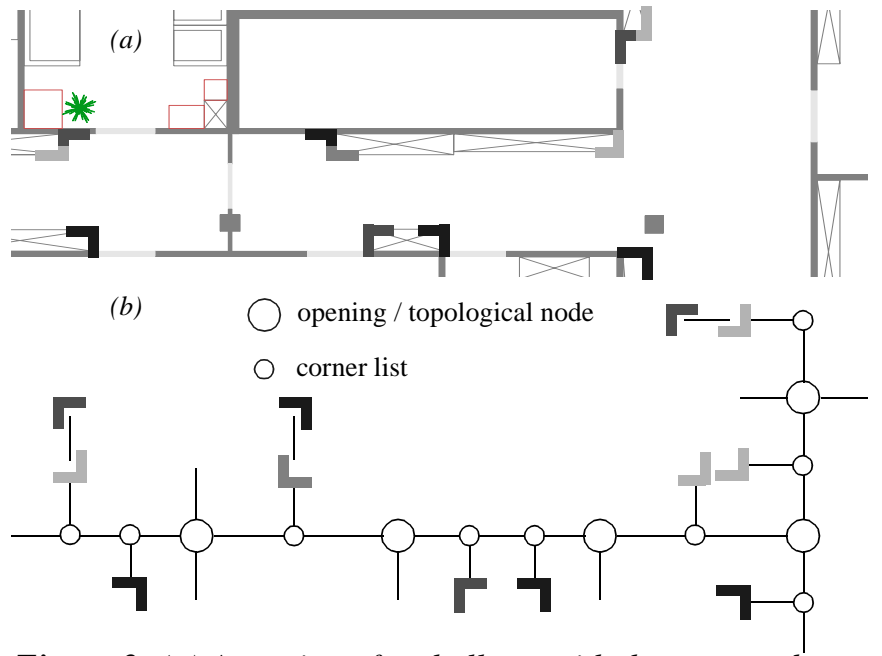

Figure 2: (a) A portion of an hallway with the extracted corner and opening features. (b) The topological map is represented by a graph.

Note that, because the sensor used is a 360 degree laser scanner, an observation contains many landmarks (typically 2 to 6 ) which are then transformed in a graph (named observation graph) compatible to the environment model.

\subsection{Local Metric Maps}

The features used for metric environmental representation are infinite lines. They are less informative than line segments, but have a better probabilistic model with analytical solution and permit a very compact representation of structured geometric environments requiring only about 10 bytes per $m^{2}$ for a typical office environment.

\section{Localization and Map Building}

The environmental models allow the use of two different navigation method with complementary characteristics. The metric localization permits a very precise positioning at the goal point [1], whereas the topological one [4] guarantees robustness against getting lost due to the multimodal representation of the robot's location. In this section the localization and map building approach is just summarized. Refer to [19] and [20] for more details. However, a detailed description of the technique used for closing the loop is presented.

\subsection{Localization and Map Building Strategy}

As explained in section 2, the environment model is com- posed of a global topological map and a set of local metric maps. Given a metric transition feature, local metric maps can be everywhere in the environment. Therefore a suitable environment-dependent strategy has to be adopted.

Being the test bed for this approach a typical office environment it can be expected that the robot will have to be very precise in rooms, where most of its tasks have to be executed (e.g. docking for recharging; manipulation of objects on a table; human-robot interaction), while in hallways robustness and global consistency take an important role. Therefore the two different levels of abstraction are used in combination of the different type of environmental structures:

- While navigating in hallways the robot firstly creates and then updates the global topological map

- When it enters a room, it creates a new local metric map

These structures are differentiated by the laser sensor. Doors represent therefore the transition between the topological and metric approach. For the initialization of the metric approach when entering a room the door is measured and used as origin of the local metric map.

\subsection{Exploration Strategy}

The proposed exploration strategy is simple: The robot first explores all the hallways in a depth-first way. It then explores each room it encountered by backtracking.

\subsection{Topological Localization and Map Building}

The environment is rectilinear and mainly composed of offices, meeting rooms and hallways. Therefore only four directions of travel are employed: N, E, S, W.

Position Estimator: Given a finite set of environment states $S$, a finite set of actions $A$ and a state transition model $T$, the model can be defined by introducing partial observability. This includes a finite set $O$ of possible observations and an observation function $O S$, mapping $S$ into a discrete probability distribution over $O . T\left(s, a, s^{\prime}\right)$ represents the probability that the environment makes a transition from state $s$ to state $s^{\prime}$ when action $a$ is taken. $O S(o, s, a)$ is the probability of making an observation $o$ in state $s$ after having taken action $a$. The probability of being in $s^{\prime}$ (belief state of $s^{\prime}$ ) after having made observation $o$ while performing action $a$ is then given by:

$$
S E_{s^{\prime}}(k+1)=\frac{O S\left(o, s^{\prime}, a\right) \sum_{s \in S} T\left(s, a, s^{\prime}\right) S E_{s}(k)}{P(o \mid a, S E(k))}
$$

where $S E_{s}(k)$ is the belief state of $s$ for the last step, $S E(k)$ is the belief state vector of last step and $P(o \mid a, S E(k))$ is a normalizing factor.

Heading Estimator: Because the position estimator does not take into account the heading of the robot, this is done separately like in [10]. However in this case the orientation is estimated by a weighted mean of each observed line that is either horizontal or vertical with respect to the environment. This can be viewed as an EKF for heading only, where no map is required because for prediction $\theta$ is directly used instead.

Control Strategy: Since it is computationally intractable to compute the optimal POMDP control strategy for a large environment [4], a simple suboptimal heuristic is introduced: 
The most likely state policy is used to find the state with the highest probability and the action that would be optimal for that state is executed. However it can happen that the robot is not sure about its current state. This is calculated by mean of the unconfident function $U(S E(k))$, which is the entropy of the probability distribution over the states of the map. The POMDP is confident when

$$
U(S E(k))=-\sum_{s} S E_{s}(k) \log S E_{s}(k)<U_{\max }
$$

where $U_{\max }$ is determined by experience. When the robot is unconfident, it follows the hallway in the direction where it expects to find more information. What has to be avoided at any cost is to switch from the multimodal topological navigation to the unimodal metric navigation when the robot is unconfident about its location, otherwise it could enter a false local metric place and therefore be lost. If such a problem occurs a solution for detecting this situation and exit the current local place would be required in order to allow the robot relocating itself by means of the topological approach.

Map Building: Instead of using a complex scheme for model learning like in [11] and [18], where an extension of the BaumWelch algorithm is adopted, here the characteristics of the $o b$ servation graph (section 2.1) are used. When the robot feels confident about its position, it can decide if an extracted landmark is new by comparing the observation graph to the node in the map corresponding to the most likely state. This can happen either in an unexplored portion of the environment or in a know portion, where new landmarks appear due to the environment dynamic. In order to cope with this dynamic each new landmark is stored in the map with a confidence value, which then rises when the landmark is re-observed and is lowered when a landmark which should have been seen is not detected. When the confidence decreases and is below a minimum, the corresponding landmark is deleted from the map. This allows for dynamics in the environment, where landmarks that disappear in the real world, will be deleted from the map too.

\subsection{Metric Localization and Map Building}

This section describes briefly the main characteristics of the stochastic map approach [16], which permits using an extended Kalman filter [7], [14] for localization.

System State Vector: With this approach both the robot position $x_{r}=(x, y, \theta)^{\prime}$ and the features $x_{i}=(\alpha, r)^{\prime}$ are represented in the system state vector:

$$
x=\left[\begin{array}{l}
x_{r} \\
x_{1} \\
x_{n}
\end{array}\right] C(x)=\left[\begin{array}{lll}
C_{r r} & C_{r 1} & C_{r n} \\
C_{1 r} & C_{11} & C_{1 n} \\
C_{n r} & C_{n 1} & C_{n n}
\end{array}\right]
$$

$C(x)$ represents the uncertain spatial relationship between objects in the map, which is changed by three actions:

- Robot displacement

- Observation of a new object

- Re-observation of an object already existing in the map
Extended Kalman Filter: When a spatial relationship is reobserved, the updated estimate is a weighted average of the two estimates calculated by means of an EKF. It permits to update a subset of the state vector while maintaining the consistency by means of the covariance matrices. A measurement equation $z=h\left(x_{1}, x . x_{m}\right)$ is considered as a function of $m$ relationships included in $x$. All of the $n$ estimates $x_{i}$ of the state vector $x$ are updated by a value that is proportional to the difference $\delta=z-\hat{z}$ between the ideal measurement $z$ and the actual measurement $z$ :

$$
\begin{gathered}
x_{i}(k+1)=x_{i}(k)+\Gamma_{i z} \Gamma_{z z}^{-1} \delta \\
\Gamma_{i z}=E\left[x_{i} \delta^{T}\right] \Gamma_{i z}=\sum_{j=1}^{M} C_{i j} H_{x j}^{T} \\
\Gamma_{z z}=E\left[\delta \delta^{T}\right] \Gamma_{z z}=\sum_{j=1}^{M} \sum_{k=1}^{M} H_{x_{j}} C_{j k} H_{x_{k}}^{T}
\end{gathered}
$$

where $H_{x_{j}}$ is the Jacobean matrix of $h$ with respect to $x_{j}$.

The variance and covariance $C_{i j}$ are also updated:

$$
C_{i j}(k+1)=C_{i j}(k)-\Gamma_{i z} \Gamma_{z z}^{-1} \Gamma_{j z}^{T}
$$

\section{Closing the Loop}

The problem of closing the loop can be defined as the question of how to know when a location has already been explored, meaning that the environment contains a loop and that the loop in the map has also to be closed.

In [18] this is achieved by adding a topological mapper which ensures global consistency. This information is then used to correct the global metric map which eventually converges to a global consistent map.

The current approach differs in two main aspects:

- Instead of closing the loops only by means of the perception, loops are detected and closed by means of the localization information.

- Loops have to be closed only in the topological map because the metric model is represented by many disconnected local metric maps.

Loops can also exist in a local metric map, however, due to the fact that these maps are supposed to be small, the drift in odometry does not cause any relevant problem to the local consistency, as it has been shown in [5].

The current method works as follows: The robot does not try to recognize if a single perception of the environment has already been seen somewhere else. However, as soon as the robot creates the map for a part of the environment which has already been visited, the probability distribution starts diverging into two peaks: One for the position in the map which is currently being created; Another for the previously created location representing the same physical place. The algorithm starts tracking the two highest probabilities as soon as the POMPD becomes unconfident because this is the first clue indicating a divergence of the probability distribution. A loop can then easily be detected when the distribution has converged into two peaks which move in the same way. The position where the loop has to be closed can then be detected by turning off the automatic mapper and backtracking with localization until the distribution re-converges to a single peak. This should also be the point where the robot started mapping the loop. A simple example is given in fig. 3 . 
(a)

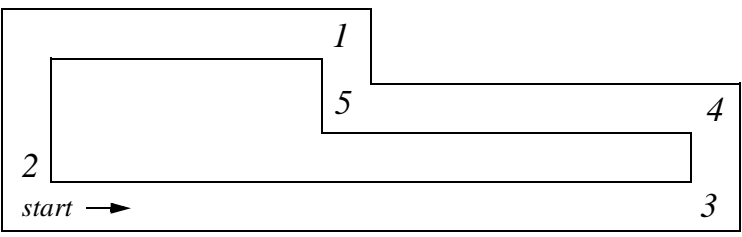

(b)

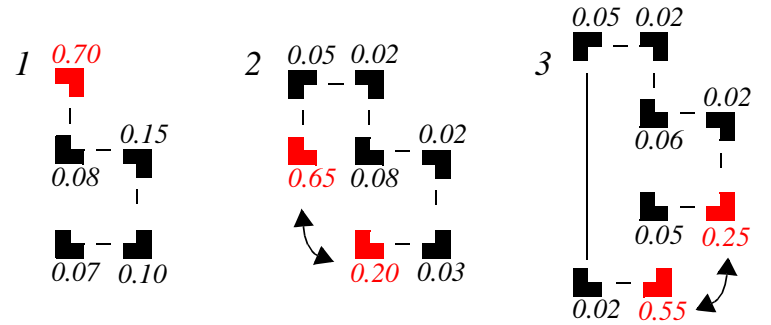

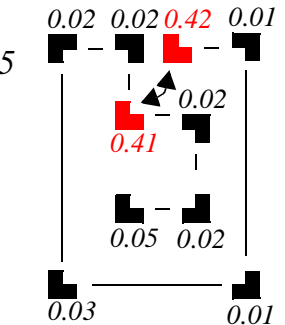

(c) backtracking

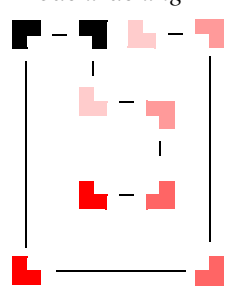

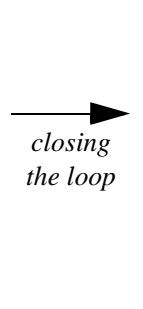

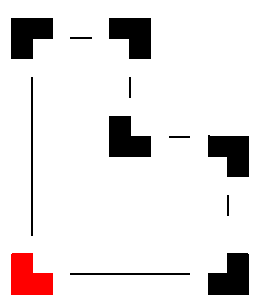

Figure 3: (a) A loop in the environment. (b) Mapping with the POMDP. 1) The map when the robot is at position 1 in the environment. 2) The robot is re-exploring the start point. The observation function $O S(o, s, a)$ gives high values for both the new node in the map and for the start node, but the probability distribution has not yet diverged because the transition function $T\left(s, a, s^{\prime}\right)$ gives a low probability of coming at the map start. 3) However by moving in the same way on the map the distribution diverges and the POMDP becomes unconfident. 4) The distribution has diverged and the two peaks move in the same way to 5. (c) The mapping is stopped. If the peaks converges at the start position by backtracking with localization, then the loop can be closed.

\section{Experimental Results}

The approach has been tested in the $50 \times 25 \mathrm{~m}^{2}$ portion of the institute building shown in fig. 4 with four different types of experiments for a total of more than $1.5 \mathrm{~km}$.

For the experiments, the mobile robot Donald Duck has been used. It is a fully autonomous mobile vehicle based on a PowerPC $604 \mathrm{e} 300 \mathrm{MHz}$ running $\mathrm{XO} / 2$, a deadline driven hard real-time operating system [3]. Donald uses encoders and SICK LMS200 laser scanners as sensors. It navigates locally by means of a motion control algorithm, which plays the role of both position controller and obstacle avoidance: It reaches the given $(x, y, \theta)$ or $(x, y)$ goal by planning a collision free path (with respect to the current local data), and reacting to the

dynamic environment either by merely replanning the path or by changing heading direction and replanning when an object appears in front of the robot.

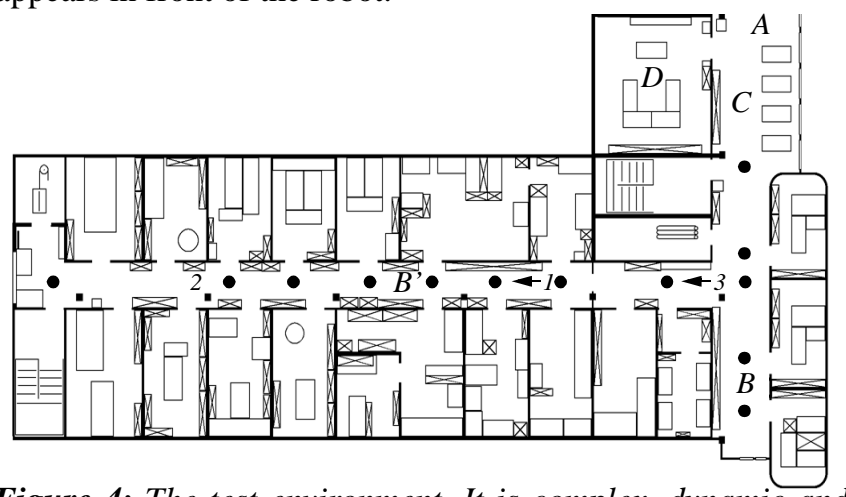

Figure 4: The test environment. It is complex, dynamic and artificially closed in A so that the exploration procedure is finite. Black dots are the places where the automatic mapper is expected to extract state nodes (the other doors are closed). In $B$ and $B$ ' the robot had problems distinguishing between the two neighbour locations. $C$ and $D$ are detected as rooms and represented by a single local metric map. A large loop does not exist in this environment. Therefore, for the experiments in section 5.3, a loop is "artificially created" by starting the exploration in 1, stopping it in 2, taking the robot manually to 3 and resuming.

\subsection{Map Building}

In this section the automatic mapping capabilities of the topological approach are tested and evaluated. Local metric maps are taken from the a priori map used in [1]. Note that the environment is arbitrarily closed (fig. 4), so that the exploration procedure is finite.

For this evaluation, five maps generated by complete explorations of the environment shown in fig. 4 are compared to evaluate their quality with respect to consistency and completeness. The results are presented in table 1 .

\begin{tabular}{|c|c|}
\hline Number of explorations & 5 \\
\hline Total travelled distance & $343 \mathrm{~m}$ \\
\hline Number of states in the environment & 13 \\
\hline Mean detected states & $12.8 / 98 \%$ \\
\hline Mean confused hallway/opening & $1.2 / 9.2 \%$ \\
\hline Mean detected features & 78 \\
\hline Mean different features & $18 / 23 \%$ \\
\hline
\end{tabular}

Table 1: Comparison of five maps generated by complete explorations of the environment shown in fig. 4.

One of the problems encountered during the exploration is the difficulty of distinguishing between opening and hallway. This leads to a mean of 1.2 false detection for each experiment. Nevertheless by visiting all the openings when traversing the environment by backtracking to add the local metric maps, these errors are detected and corrected. In one experiment a state (opening) was not extracted at all.

For the corner features the difference between two maps is analyzed. The mean amount of extracted corners in a map is 78 ; an average of 18 of these are noisy features that are not always extracted. This means that almost $77 \%$ of the features 
are constant in the five maps showing that the perception delivers valuable information to the mapper.

\subsection{Localization}

The quality of a map can also easily be estimated by testing it for localization. For this two types of localization experiments are performed: One for localization (position tracking) and the other for relocation.

To test the topological localization, 25 randomly generated test missions for a total of about $900 \mathrm{~m}$ and 28000 estimates are performed. The robot knows in which state it is at the start point. A mission is successful when the robot reaches its goal location, is in front of the opening and is confident about its position. For a precise analysis each state transition is stored in a $\log$ file with all the information, permitting to know if each state transition detected by the localization took place physically. The results are presented in table 2. Even if all the missions are successful the log file permits to detects 21 false state transitions that caused 404 false estimates in $B$ and $B$ ' (fig. 4), where the peak probability moved forward and backward between two neighbor states. These false estimates represent only $1.4 \%$ of the total, meaning that the system recovers quite fast from these errors. Nevertheless the robot had also confident false estimates $(0.5 \%)$ that can cause a mission failure if the goal state is estimated when the robot is in front of a another opening.

\begin{tabular}{|c|c|}
\hline Number of missions & 25 \\
\hline Success & $25 / 100 \%$ \\
\hline Total travelled distance & $899 \mathrm{~m}$ \\
\hline Mean travel speed & $0.31 \mathrm{~m} / \mathrm{s}$ \\
\hline Total estimates & 27870 \\
\hline Unconfident states & $3413 / 12 \%$ \\
\hline False estimates & $404 / 1.4 \%$ \\
\hline Confident false estimates & $149 / 0.5 \%$ \\
\hline
\end{tabular}

Table 2: Localization experiments. All the test missions have been successfully performed. However the robot also made false state transitions that caused some false estimates (1.4\%). This happened only by $B$ and $B$ ' in fig. 4. The reason that lead to a success rate of $100 \%$ is that the system always recovered from its error without estimating the goal location in front of a false opening. Nevertheless the robot had also confident false estimates $(0.5 \%)$ that could cause mission failure.

The second type of test is focused on recovering from a lost situation (relocation). Ten experiments are started from a randomly defined position in the environment with an overall constant belief state (i.e. lost situation). In table 3 the tests are resumed briefly.

As expected the robot can always recover. However, the system requires a minimum of 1 and a maximum of 4 states to recover. The interesting point is that this difference in the results is position dependent and repeatable. For example the crossing between the two hallways permits to recover with a single state because it is global distinctive for the environment in fig. 4. On the other hand, the right part of the horizontal hallway seems to be more distinctive than the left one where the robot require the maximum amount of states to recover.

\begin{tabular}{|c|c|}
\hline Number of experiments & 10 \\
\hline Total travelled distance & $250 \mathrm{~m}$ \\
\hline Mean distance for recovering & $13.7 \mathrm{~m}$ \\
\hline Min / max distance for recovering & $1.21 / 20.31 \mathrm{~m}$ \\
\hline Mean number of state for recovering & 2.11 \\
\hline Min / max state for recovering & $1 / 4$ \\
\hline
\end{tabular}

Table 3: Recovering from a lost situation (i.e. overall constant belief state). The robot requires from 1 to 4 states to recover, depending on the distinctiveness of the part of the environment where it is moving.

\subsection{Closing the Loop}

In the test environment there are no large loops. In order to test the proposed approach, a loop is artificially created by displacing the robot during the exploration as shown in fig. 4 . This experiment has been performed three times. Each time the probability distribution has effectively diverged into two peaks allowing to detect the loop. In order to close the loop the robot has gone back until the distribution has converged to a single confident peak. This took place where the map has been started ( 1 in fig. 4 ) proving that the loop could be closed correctly.

\section{Related Work}

Simultaneous localization and map building research can be divided into two main categories: Metric and topological. Metric approaches are defined here as methods, which permit the robot to estimate its $(x, y, \theta)$ position, while topological are those where the position is given by a location without metric information.

After the first precise mathematical definition of the stochastic map [16], early experiments [7], [14], have shown the quality of fully metric simultaneous localization and map building with respect to precision. However their heavy reliance on odometry makes the global consistency of the map difficult to maintain in large environments. Furthermore they represent the robot position with a single Gaussian distribution meaning that an unmodeled event (i.e. collision) could cause a divergence between the ground truth and the estimate from which the system is unable to recover (lost situation). In [5] it has been shown that by taking into account all the correlations the global consistency is better maintained, but this is not sufficient, as confirmed by a recent work [6], where a solution is proposed by extending the absolute localization to include a localization relative to local reference frames. Some recent works, such as [2], propose Multi Hypotheses Tracking (MHT) with EKFs. These approaches are then precise and robust. However, when the robot has to relocalize itself, localization during motion is infeasible due to the computational complexity of this task. Furthermore, the question of how to deal with this kind of multi hypotheses when planning remains open.

On the other hand topological approaches [12] can handle multi hypotheses tracking and have a topological global consistency, which is easier to maintain. Their robustness has been proven firstly with the state set progression [15], which 
has then been generalized to the POMDP approach [4], [10]. For automatic mapping in [11] the Baum-Welch algorithm has been used for model learning. In contrast to the above mentioned topological approaches, [13] proposes a topological approach, which heavily rely on odometry in order better to handle dynamic environments. All these approaches are robust, but are imprecise because the position is represented by a location without precise metric information. To face this, the Markov localization [9] has been proposed: A fine grained grid guarantees both precision and multimodality. However this approach remains computationally intractable for current embedded systems. A more efficient alternative has recently been proposed, but the Monte Carlo localization [8] has not yet been extended for map building.

Metric and topological approaches are converging, as in [6], [8] and [9], to hybrid solutions. Going in this direction, in [17] the approach consists in extracting a topological map from a grid map by means of a Voronoi based method, while [18] proposes to use the Baum-Welch algorithm as in [11], but to build a topologically consistent global map which permits closing the loop for the global metric map too.

In contrast to the above mentioned approaches, for this system a natural integration of the metric and topological paradigm is proposed. The approaches are completely separated into two levels of abstraction. Metric maps are used only locally for structures (rooms) that are naturally defined by the environment. The topological approach is used to connect the local metric maps that can be far away from each other. With this the robot can take advantage of the precision of a fully metric, EKF navigation, added to the robustness in the large of the POMDP approach. Furthermore the approach allows to coherently close loops in the environment.

\section{Conclusions and Outlook}

This paper presents a hybrid approach for both localization and map building. This combination permits both precision with the non-discrete metric estimator and robustness by means of the multi hypotheses topological approach.

The approach is validated empirically by extensive experimentation for a total of more than $1.5 \mathrm{~km}$. Map building is tested by performing five complete explorations of the environment and comparing the resulting maps. This comparison demonstrates that the maps are consistent with respect to the environment and that the perception permits to extract precious information. For localization, the success rate over the $0.9 \mathrm{~km}$ of the 25 tests missions is $100 \%$. Nevertheless a precise analysis of the state transitions shows that, between neighbor states, false state estimate occurs $(1.4 \%)$ and sometimes are even treated as confident $(0.5 \%)$. The relocation performance of the topological method has been shown with 10 successful experiments where the belief state converges with 1 to 4 states depending on the distinctiveness of the part of the environment where the robot is navigating. Moreover it has been shown how loops can be closed on the localization level instead of the perception level. This is done within the same compact framework by the multi hypotheses tracker of the POMDP for detection and backtracking for closing the loop.

\section{Acknowledgements}

Thanks to Gilles Caprari and Marc de Battista at ASL, for their valuable feedback about this work.

\section{References}

[1] Arras, K. O., N. Tomatis, et al. (2001). "Multisensor On-theFly Localization: Precision and Reliability for Applications". Robotics and Autonomous Systems 34(2-3).

[2] Arras, K. O., J. A. Castellanos, et al. (2002). Feature-Based Multi-Hypothesis Localization and Tracking for Mobile Robots Using Geometric Constraints. IEEE International Conference on Robotics and Automation, Washington DC.

[3] Brega, R., N. Tomatis, et al. (2000). The Need for Autonomy and Real-Time in Mobile Robotics: A Case Study of XO/2 and Pygmalion. IEEE/RSJ International Conference on Intelligent Robots and Systems, Takamatsu, Japan.

[4] Cassandra, A. R., L. P. Kaelbling, et al. (1996). Acting under Uncertainty: Discrete Bayesian Models for Mobile-Robot Navigation. IEEE International Conference on Robotics and Automation, Osaka, Japan.

[5] Castellanos, J. A., J. D. Tardós, et al. (1997). Building a Global Map of the Environment of a Mobile Robot: The Importance of Correlations. IEEE International Conference on Robotics \& Automation, Albuquerque.

[6] Castellanos, J. A., M. Devy, et al. (2000). Simultaneous Localization and Map Building for Mobile Robots: A Landmarkbased Approach. IEEE International Conference on Robotics and Automation, San Francisco.

[7] Crowley, J.L. (1989). World Modeling and Position Estimation for a Mobile Robot Using Ultrasonic Ranging, IEEE International Conference on Robotics and Automation, Scottsdale, AZ.

[8] Dellaert F., D. Fox et al. (1999). Monte Carlo Localization for Mobile Robots, IEEE International Conference on Robotics and Automation, Detroit Michigan.

[9] Fox, D. (1998). Markov Localization: A Probabilistic Framework for Mobile Robot Localization and Navigation. Institute of Computer Science III. Bonn, Germany, University of Bonn.

[10] Gutierrez-Osuna, R. and R. C. Luo (1996). "LOLA: probabilistic navigation for topological maps." AI Magazine 17(1).

[11] Koenig S., R.Goodwin, et al. (1995). Robot Navigation with Markov Models: A Framework for Path Planning and Learning with Limited Computational Resources. International Workshop, Reasoning with Uncertainty in Robotics, RUR '95, Amsterdam, Netherlands, Springer.

[12] Kuipers, B. J. and Y. T. Byun (1987). A qualitative approach to robot exploration and map-learning. Workshop on Spatial Reasoning and Multi-Sensor Fusion, Los Altos, CA, USA, Morgan Kaufmann.

[13] Kunz, C., T. Willeke et al. (1999). “Automatic Mapping of Dynamic Office Environments.” Autonomous Robots 7.

[14] Leonard, J.J., H.F. Durrant-Whyte, et al. (1992). "Dynamic Map Building for an Autonomous Mobile Robot", The International Journal of Robotics Research 11(4).

[15] Nourbakhsh, I. (1998). Dervish: An Office-Navigating Robot. Artificial Intelligence and Mobile Robots. D. Kortenkamp, R. P. Bonasso and R. Murphy, The AAAI Press/ The MIT Press: 73-90.

[16] Smith R. C., M. Self, et al. (1988). Estimating uncertain spatial relationships in robotics. Uncertainty in Artificial Intelligence 2. J. F. Lemmer and L. N. Kanal, Elsevier Science: 435-461.

[17] Thrun, S. and A. Bücken (1996). Integrating grid-based and topological maps for mobile robot navigation. National Conference on Artificial Intelligence, AAAI, Portland, USA.

[18] Thrun, S., J.-S. Gutmann, et al. (1998). Integrating Topological and Metric Maps for Mobile Robot Navigation: A Statistical Approach. Tenth Conference on Innovative Applications of Artificial Intelligence, Madison, WI.

[19] Tomatis, N., I. Nourbakhsh, et al. (2001). A Hybrid Approach for Robust and Precise Mobile Robot Navigation with Compact Environment Modeling. IEEE International Conference on Robotics and Automation, Seoul, Korea.

[20] Tomatis, N., I. Nourbakhsh, et al. (2001). Simultaneous Localization and Map Building: A Global Topological Model with Local Metric Maps. IEEE/RSJ International Conference on Intelligent Robots and Systems, Maui, Hawaii. 